\title{
Multi-function vehicle and control method for intelligent home security environment monitoring
}

\author{
Yang Chen, Yanni Deng, Wenchao Xiao \& Fan Hao \\ School of Automation, Wuhan University of Technology, Wuhan, China
}

\begin{abstract}
With the development of science and technology and the improvement of living standards, people in ensuring the precursor of family safety on quality of life and intelligent family life increasingly high expectations. But the quality of life only in terms of temperature and humidity real-time monitoring, not only the need for wiring large quantities, high cost for each room. Good health and long down the electromagnetic pollution on people also have some impact. And all the data collected are independent not comprehensive decision analysis, intelligent degree is relatively low. And in the current increasingly serious air pollution at the same time, temperature and humidity measurement of traditional can not meet the demand for air quality concerns. The traditional camera installation position is fixed, there is a blind spot monitoring caused high-rise tenants theft incidents have also occurred frequently. This paper presents a family of intelligent car And its control method, low cost, high degree of intelligence, not only can real-time monitoring of the state of the environment, but also can be transmitted back to the video screen in real time.
\end{abstract}

KEYWORD: control, intelligence, air quality concerns, low cost, video screen

\section{CAR COMPOSITION AND SPECIFIC MODULES}

The top view and the positive view of the car are shown in Figure 1, figure 2:

Figure parts respectively: 1- camera 2- infrared thermal imager 3- DC motor 4-Infrared tracking of tube 5- air quality detector 6- car body 7- mechanical arm 8- power supply system 9- wireless communication module 10- voice alarm device 11- ultrasonic sensor.

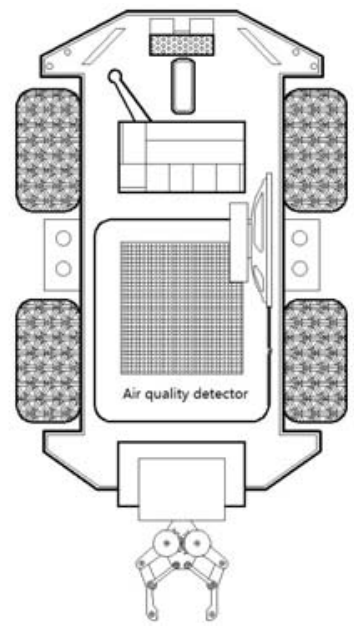

Figure 1 top view of car

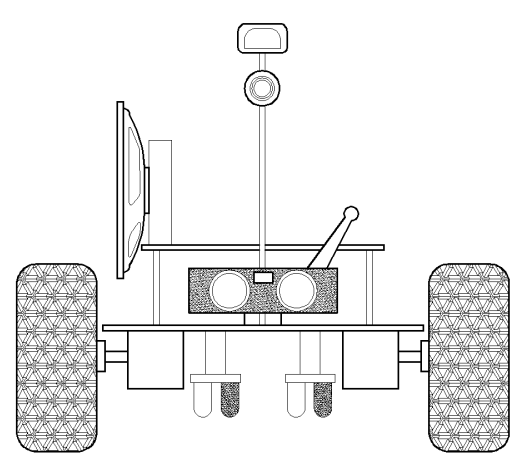

Figure 2 positive view of car

\subsection{Driving module}

The car using driven $293 \mathrm{~d}$ and $293 \mathrm{~d}$ is an $\mathrm{H}$ bridge driving circuit, very convenient to control the motor positive inversion, driving ability for this work enough to provide $0.6 \mathrm{~A}$ each bridge (1.2A peak) with overheat protection, voltage from $4.5 \mathrm{~V}$ to $36 \mathrm{~V}$ optional, connecting two channels of $5 \mathrm{~V}$ simple steering gear, the 4-way bi-directional DC motor connected, two stepper motor is connected, very suitable for high integration requires the use of.. The driving circuit is shown in Figure 3. 


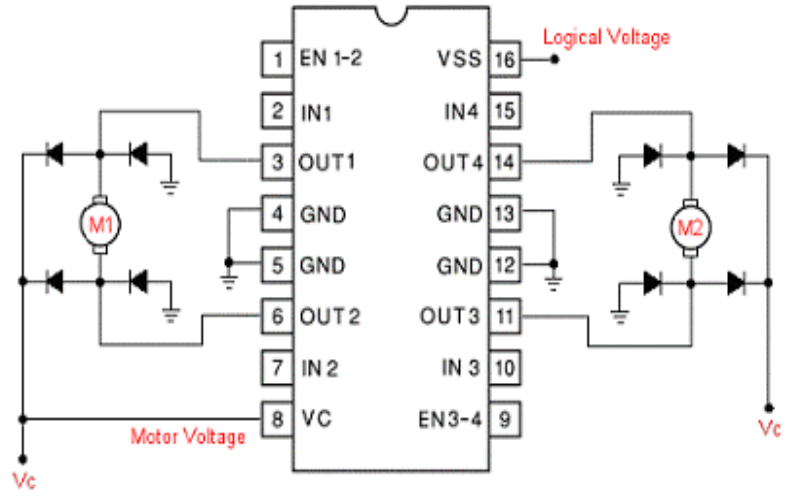

Figure 3 driving circuit

\subsection{Manipulator arm module}

Mechanical arm is depended on the actuator to work, is composed of two joints and a plurality of gears and other hardware structure, can realize rotation and about open and closed, the actuator is the hardware core of mechanical arm to be aided software, to achieve a simple clamping function

\subsection{Voice alarm module}

Isd1700 series chip is Winbond Company launched the monolithic quality voice recording circuit, the chip provides a number of new features, including built-in patent information management system, the new information prompt, the dual operation mode of independent Principle as shown in Figure 4.

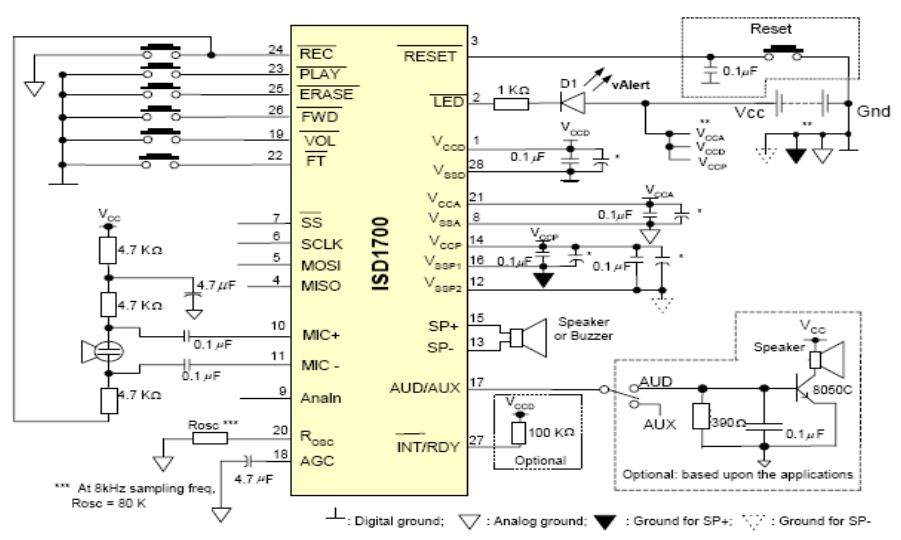

Figure 4 dual operation mode of independent Principle

\subsection{Obstacle avoidance module introduction}

The sensor module of the light environment adaptability, it has a pair of infrared transmitting and receiving tube, tube launched a certain frequency of infrared detection, when the obstacle direction (reflector), reflected infrared receiving tube, the comparator circuit after processing, the green LED lights, and digital output the signal output interface signal (a low signal), can adjust the detection distance through the potentiometer knob, the effective distance range of $2 \sim 30 \mathrm{~cm}$, the working voltage of the sensor for detecting the $3.3 \mathrm{~V}-5 \mathrm{~V}$. Distance can be adjusted by a potentiometer, with little interference, easy assembly, easy to use, can be widely used in robot obstacle avoidance, obstacle avoidance car, line count and the black and white line tracking and other occasions. Principle as shown in Figure 5

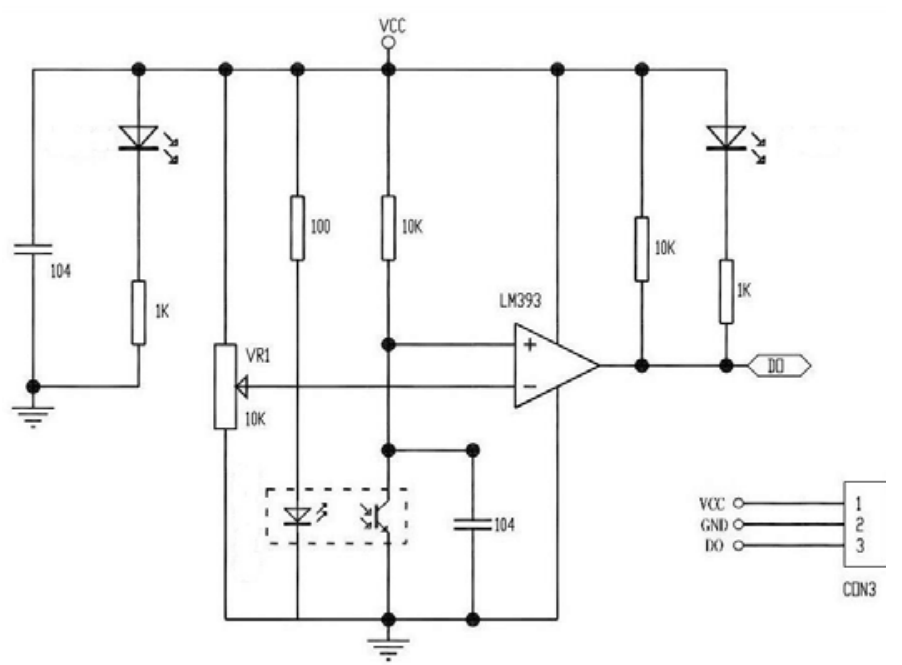

Figure 5 Principle

\section{TROLLEY AND MAIN FUNCTION INTRODUCTION}

In other embodiments of this, the invention implementation example of intelligent family car with general intelligent car body, four wheel with to DC motor, the front is equipped with two degrees of freedom of the camera and infrared thermal image instrument. Front mounted four infrared tracking on the tube. Installed in front of the ultrasound barrier module. Vehicle equipped with Android or IOS device control software of the controller. Body is located in the middle of the air quality detection instrument. The tail end of the car for car power system, voice broadcast device and two degree of freedom manipulator. Car by identifying the user laying good route, running.

Camera, an infrared camera: car have a day and night modes. Open camera model, infrared camera off. Trolley moving through the camera, down, left and right swing on the family environment for realtime monitoring. In the night mode is turned on the infrared camera, close the camera. The car key to the living room balcony, and other parts of the heat source monitoring. HD camera has two degrees of freedom can sway, real-time transmission of family picture to the mobile terminal. The body place of 180 DEG jerked backward supervision. Infrared thermal imager can at night specific windows and doors accurately capture the images of the human body, so as to identify the risk. 
Infrared tracking through the identification of laying track, determine the car driving will not deviate from the track. Android, IOS control software, through wireless means, such as WiFi or Bluetooth, the car and the mobile terminal is connected and remote communication, remote control car. Users only need to download a specific APP in the mobile terminal. Air quality detector not only on the environmental temperature and humidity for real-time detection of PM2.5 PM10 concentration of major pollutants in the atmosphere is always measured. The real-time detection of temperature and humidity air quality detector, air quality testing and displayed by the LCD screen. The alarm when the temperature is higher than 75 degrees or less than 0 degrees, also issued a warning when the family of air quality pollution or flue gas concentration is too high. The car body is made of alloy material, high strength, good effect of load power supply system using wired and wireless charging two modes, with a voltage stabilizing circuit and charge the discharge protection circuit. When the detected temperature is too high, the night alarm danger and the concentration of pollutants exceed the standard, the voice module play good storage content. Alarm equipment recorded voice information preset, when an emergency will be issued when the specific speech to prompt the risk prompt buzzer sounds. Mechanical arm has 2 degrees of freedom. Can carry on some minor items. The front of the car is equipped with ultrasonic barrier device, when there is an obstacle on the track when the car, the car will stop a specified tone. Air quality detector car equipped with LCD screen on the face can clearly demonstrate a variety of parameters in the room or by the current. The speech parameters, improve the quality of life.

\section{FACE RECOGNITION CONTROL METHOD}

In the control of face recognition is different with the traditional method. Cameras and infrared thermal imager are traditional methods to identify the face image. And simple face recognition using the camera or infrared imager, has its advantages but also have the corresponding defects and deficiencies. The simple use of camera for face recognition the input is characteristic of pixel, the whole face image, ignoring the local image features, local features make it difficult to record and face. The face is a non rigid feature, when the facial expression changes, each organ facial deformation will occur in different degrees. And in the admission conditions, usually made of face image by the light, background and other factors in different degree, it is difficult to distinguish between the facial organs in the face recognition plays a larger role, what role is small. But the benefits are withdrawn Face image with high resolution image is clear. Therefore, the simple use of camera extracted from face images, the overall im- age with high resolution, but local feature is not obvious. Only using infrared thermal imager for face recognition, subject to the development of the infrared thermal imager, infrared thermal imaging system resolution is not high, resulting in the extraction of image resolution is not high. But the infrared thermal imager to temperature sensitive, accurately extract the features of face organs.

Feature vector of HOG after fusion $\theta \mathrm{i}$. To determine the classification and face recognition and then determine the label support vector machine on each dimension vector in $\mathrm{n}$-dimensional $\mathrm{m}$.

Method of control method on face recognition using the camera and infrared camera combination, improve the face recognition rate, can accurately identify the stranger. The face recognition algorithm of face image recognition, the first member of the family, which is stored in the internal memory module. While on patrol, work through the camera and infrared thermal imager, face recognition of the collected image, face image and recognition results will be compared with family members, if the face image feature information with family members, it is judged as the security situation, do not send warning information; if the face image feature information of family members do not meet, it is judged as invasion of foreign workers the status and alarm information.

4. The control system of intelligent home security environment monitoring multi function car is shown in figure 6.

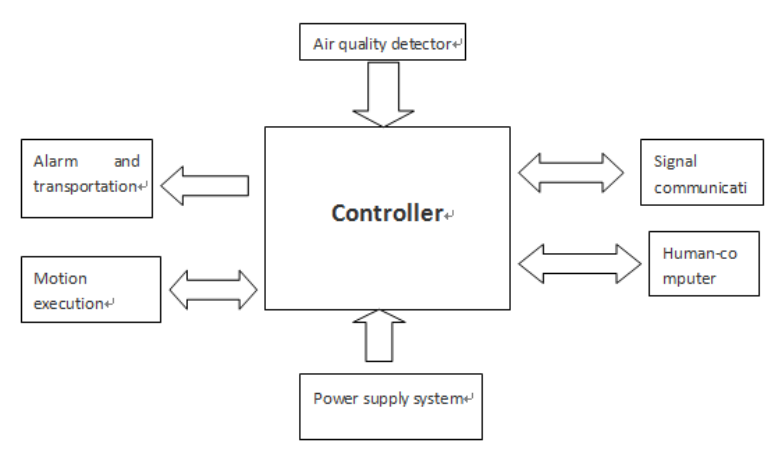

Figure 6 control system of intelligent home security environment monitoring multi function car

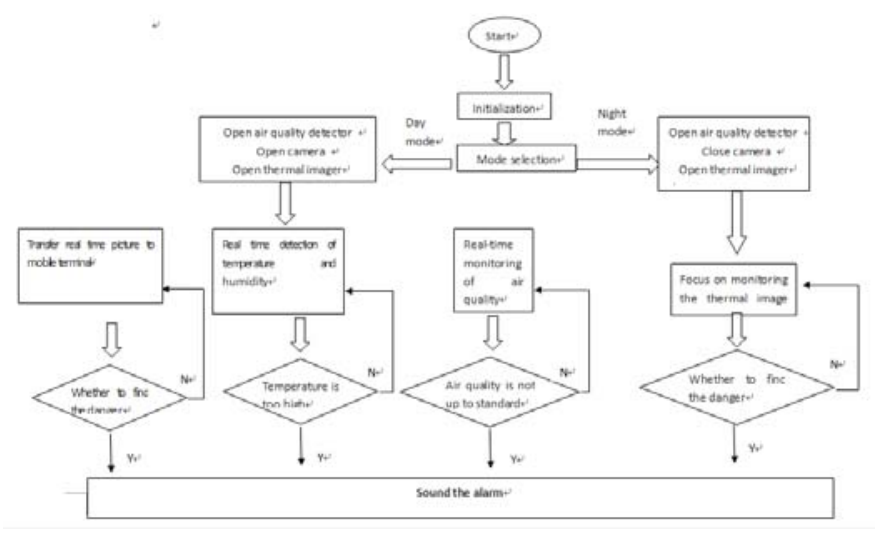

Figure 7 Control method of the multi function car 
5. The control method of the multi function car for the intelligent home security environment monitoring is shown in figure 7 ;

\section{CONCLUSIONS}

The mentioned in the smart car based on existing technology in home security cameras installed fixed position, and the defects of single function provides a can be easily moved, and rich in monitoring function of smart home burglar environment monitoring car and face recognition control method. In solving the comprehensive air quality monitoring, real time information acquisition and real-time decision making and traditional cameras for home surveillance and high cost of the three main problems has been the outstanding improvement of the comprehensive utilization of, effective intelligent home and quality are proud to improve.

\section{REFERENCES}

F.-Y.Wang, X.Wang, and L.Li, etal., "Design and Construction of a Digital Vehicle Proving Ground," IEEE Intelligence System, 2003. Vol.18, (2), 12-15.

Hamada Alshaer, Thierry Ernst. "An Integrated Architecture for Multi-homed Vehicle-to-Infrastructure Communications" 2012 IEEE Network Operations and Management Symposium pp1042-1047

Liu Lianhao "A new street lamp controller design" Computing Technology and Automation 1997 p61-63

L.Li and F.-Y.Wang, "Vehicle trajectory generation for optimal driving guidance," Proceedings of the IEEE 5th International Conference on Intelligent Transportation Systems, 2002, pp.231-235.

WU Yue, SHI Changhong. "Design of New Intelligent Street Light Control System" IEEE International Conference on Control and Automation 2010 ThCP4.16

$\mathrm{Xu}$ Kaihua, Wang Ya."Design and Implementation of Intelligent Vehicle Monitoring and Management System Based on the Multi-Net" IEEE Asia-Pacific Conference on Services Computing 2006 APSCC'06

$\mathrm{Xu}$ Kaihua, Liu Yuhua, “A Novel Intelligent Transportation Monitoring and Management System Based on GPRS", The Proceedings of 2003 IEEE International Conference on Intelligent Transportation System-Vol.2, 2003 